Gustavo Avila-Ortiz

Rodrigo Neiva

Pablo Galindo-Moreno

Ivan Rudek

Erika Benavides

Hom-Lay Wang

\title{
Analysis of the influence of residual alveolar bone height on sinus augmen- tation outcomes
}

\section{Authors' affiliations:}

Gustavo Avila-Ortiz, Pablo Galindo-Moreno, Ivan

Rudek, Erika Benavides, Hom-Lay Wang,

Department of Periodontics and Oral Medicine,

University of Michigan School of Dentistry, Ann

Arbor, MI, USA

Rodrigo Neiva, Department of Periodontology,

University of Florida College of Dentistry,

Gainesville, FL, USA

Pablo Galindo-Moreno, Department of Oral Surgery and Implant Dentistry, University of Granada

School of Dentistry, Granada, Spain

\section{Corresponding author:}

Gustavo Avila-Ortiz

Department of Periodontics and Oral Medicine

University of Michigan School of Dentistry

1101 N. University, Ann Arbor, MI 48109-1078

Tel.: 7347633325

Fax: 7349360374

e-mail: avila@umich.edu

Key words: bone regeneration, bone substitutes, clinical research, clinical trials, guided tissue regeneration, sinus floor elevation

\begin{abstract}
Introduction: Maxillary sinus augmentation is a predictable implant site development technique for posterior atrophic maxillary ridges. However, graft consolidation requires adequate angiogenesis and migration of osteogenic cells from native bone. Therefore, the amount of residual bone height $(\mathrm{RBH})$ may play a role in the rate of graft maturation. The purpose of this study was to analyze the influence of RBH in the histomorphometric outcomes of maxillary sinus augmentation procedures.

Material and methods: Patients in need of sinus augmentation were recruited for the study. Customized radiographic guides were fabricated and a cone-beam computerized tomography scan was obtained at baseline. Two examiners measured RBH on the scans at the locations marked by the radiographic guide. Sinus grafting was performed by a lateral window approach using a particulated mineralized allograft. Patients were followed up for 6 months. At the time of implant placement, bone core biopsies were harvested using the radiographic guide, which was converted into a surgical guide. Samples were histomorphometrically analyzed. Proportion of vital bone (\% VB), remaining allograft particles (\%RA), and non-mineralized tissue ( $\% \mathrm{NMT})$ were quantified. Categorical analysis of correlation of RBH ( $<4$ or $\geq 4 \mathrm{~mm}$ ) with\%VB and\%RA was performed using a statistical model.

Results: Twenty-one patients underwent sinus augmentation for a total of 21 sinuses. One patient developed an infection after grafting and was excluded. Histomorphometric analysis revealed that mean $\%$ VB was $20.47 \pm 18.25$, mean $\%$ RA was $29.04 \pm 24.94$, and average $\%$ NMT was $50.47 \pm 12.76$. No significant correlation between RBH and $\% \mathrm{VB}(r=0.016 ; P=0.951)$, and RBH and $\%(r=0.009 ; P=0.971)$ was found. Similarly, categorical analysis of correlation showed no statistical significance.

Conclusion: These findings suggest that the remaining alveolar bone height does not appear to influence the maturation and consolidation of an allograft in the maxillary sinus.
\end{abstract}

Residual alveolar bone height $(\mathrm{RBH})$ is a critical anatomic factor that is carefully considered when planning a maxillary sinus augmentation procedure. Given its direct influence on implant primary stability, $\mathrm{RBH}$ is commonly used to determine the implant placement protocol of choice, either simultaneous or delayed. Abundant and compact residual alveolar bone favors implant primary stability. Conversely, achieving primary stability is often challenging in sites exhibiting limited and coarse alveolar bone. In the vast majority of maxillary sinus augmentation protocols, the cut-off value to discern whether to perform simultaneous or delayed implant placement is in the range of 4 to 6 mm of RBH (Wang \& Katranji 2008; Misch et al. 2009; Nkenke \& Stelzle 2009). Interestingly, high implant survival rates have been extensively reported in challenging clinical scenarios, where implants were simultaneously placed with $<5 \mathrm{~mm}$ of $\mathrm{RBH}$ (Peleg et al. 1998, 1999, 2006; Rodriguez et al. 2003; Mardinger et al. 2007). Therefore, the impact of RBH on implant stability and survival has been the subject of investigation (Fenner et al. 2009a,b; Rios et al. 2009; Urban \& Lozada 2010). However, the importance of RBH 
is not exclusively related to implant stability and implant success/survival rates. The success of any bone grafting procedure, including maxillary sinus augmentation, relies on the provision of adequate angiogenesis and migration of osteogenic cells from native bone (Busenlechner et al. 2009). Therefore, it has been suggested and it can be hypothesized that residual alveolar bone, as a foundational source of cells and blood supply, may play an important role in the consolidation and maturation of a maxillary sinus graft.

This case series study was aimed at evaluating the influence of $\mathrm{RBH}$ on histomorphometric outcomes, such as proportion of vital bone $(\% \mathrm{VB})$ and remaining allograft particles (\%RA), following sinus augmentation using an allogenic grafting material.

\section{Material and methods}

\section{Patients}

All participants in the study were recruited and treated in the Graduate Periodontics Clinic at the University of Michigan School of Dentistry. The University of Michigan Institutional Review Board approved the experimental protocol (HUM00017520). The study was also registered in the database of the National Institutes of Health (NIH) (http://www.clinicaltrials.gov) under the identification number 'NCT00868777'. Adult patients between 18 and 85 years of age, in need of unilateral or bilateral sinus augmentation with delayed implant placement, presenting physical status according to the American Society of Anesthesiologists (ASA) of I or II (http://www.asahq.org/clinical/physicalstatus.htm), exhibiting $\mathrm{RBH} \leq 6 \mathrm{~mm}$ (assessed in periapical radiographs taken with the paralleling technique), and O'Leary plaque score $\leq 20 \%$ (O'Leary et al. 1972) were included in the study. Patients were excluded from the study if reported long-term ( $>2$ weeks) use of antibiotics within the previous 3 months, use of medications known to affect bone metabolism, uncontrolled conditions known to alter bone metabolism, smoking more than 10 cigarettes per day (Levin et al. 2004), history of alcoholism or recreational drug abuse, mucocutaneous diseases, severe acute or chronic sinus pathology (i.e. sarcoidosis, osteomas, carcinomas), history of cancer, radiation to the head and neck in the last 18 months, and chemotherapy in the last 12 months or postoperative complications related to these therapies. Female patients who were pregnant or attempting to get pregnant at the time of screening were also excluded. Prior to inclusion in the study, patients were required to read, understand, and sign an informed consent form.

\section{Surgical planning}

Stone models of all patients were obtained from upper and lower alginate impressions. Customized radiographic guides were fabricated, as described elsewhere (Avila et al. 2010b). Cone-beam computed tomography (CBCT) scans were obtained from each patient using an i-CAT cone-beam machine (Imaging Sciences International, Inc, Hatfield, PA, USA). Only the maxilla and maxillary sinuses were scanned in these patients to minimize the radiation exposure unless a request to include the mandible or other areas of the skull in the same scan was made for other purposes not related to this research study. The Field of View (FOV) used was $6 \mathrm{~cm}$ for the maxilla and maxillary sinuses only, $8 \mathrm{~cm}$ when both jaws were included in the scan, and $13 \mathrm{~cm}$ for the entire head. The machine settings are fixed at $120 \mathrm{kVp}$ and $18.66 \mathrm{mAs}$ for all scans regardless of the FOV used. Linear measurements to determine the $\mathrm{RBH}$ were made using a radiopaque cylindrical marker
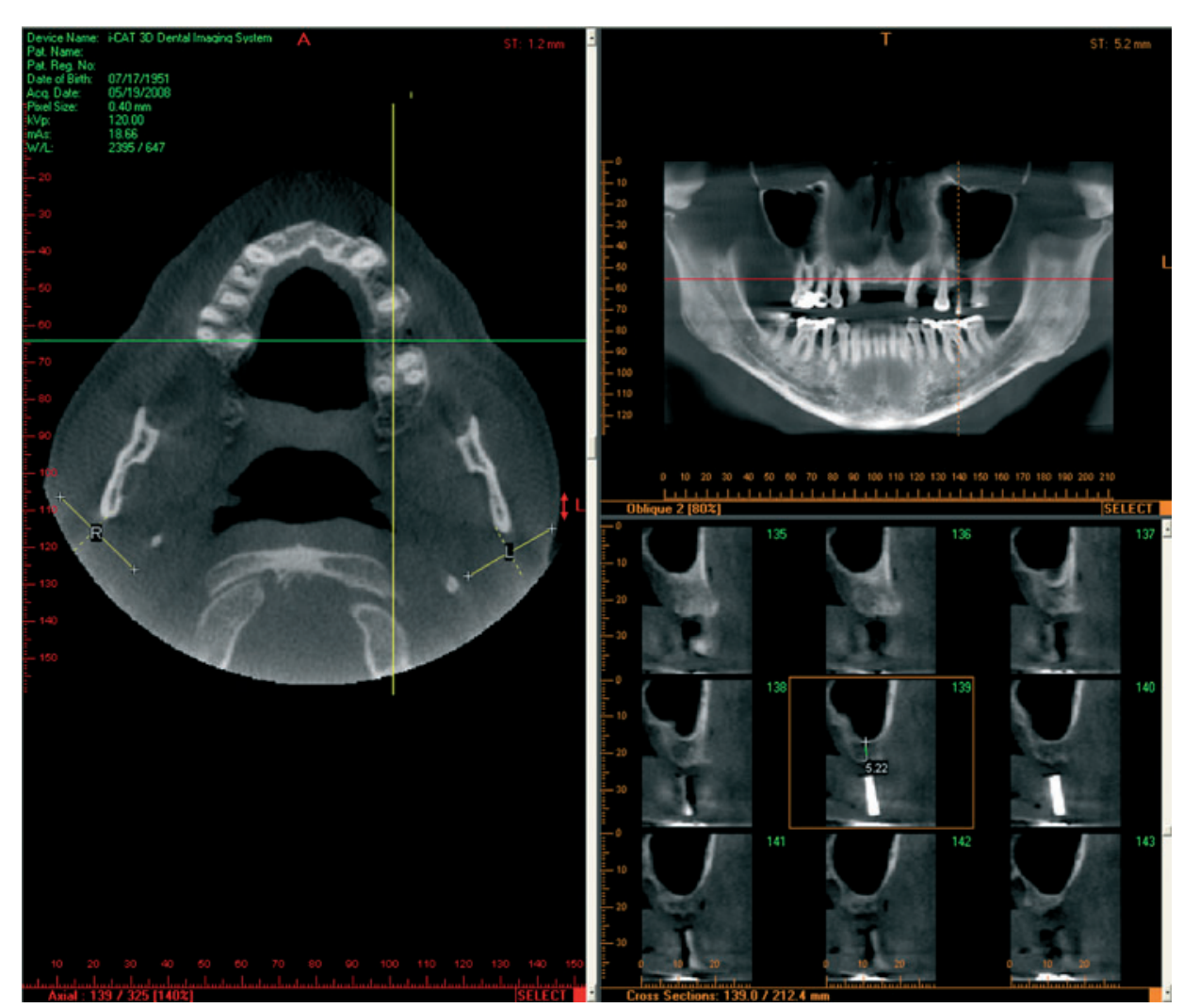

Fig. 1. Linear measurements using the radiopaque marker of the guide as a reference were performed to assess the residual bone height $(\mathrm{RBH})$.

embedded in the guide as reference (Fig. 1). Proprietary software provided by the manufacturer of the scanner was used for these measurements (iCAT; Xoran Technologies Inc., Ann Arbor, MI, USA). Two calibrated, blinded examiners specifically trained for this project performed the measurements twice. Measurements were averaged and expressed as the mean value between the average of the two sets of measurements that were independently obtained by each examiner.

All participants were asked to follow a pharmacologic protocol consisting of antibiotics (Amoxicillin $500 \mathrm{mg}$ TID for 10 days, starting 2 days before the surgery; or clindamycin $300 \mathrm{mg}$ TID for 10 days, starting 2 days before the surgery, for patients allergic to penicillins) and oral corticosteroids (Dexamethasone $8 \mathrm{mg}$ QD, $24 \mathrm{~h}$ before the surgery) to control postoperative swelling and discomfort.

\section{Maxillary sinus augmentation}

Surgeries were performed under intravenous sedation and local anesthesia (xylocaine 2\% $1: 100,000$ or $1: 50,000$; Astra Zeneca USA, Inc., Pharmaceuticals, Westborough, MA, USA). In all cases, sinus augmentation was performed following a lateral approach.

(C) 2011 John Wiley \& Sons A/S 
Briefly, a supracrestal incision was made on the edentulous segment, with vertical releases if needed to facilitate surgical access. Following full-thickness flap elevation, a lateral window was prepared with a round diamond bur. After exposure of the Schneiderian membrane, specifically designed hand instruments were used for its elevation (Salvin Dental Specialties, Inc., Charlotte, NC, USA). A mineralized bone allograft with particle size ranging from 600 to $1250 \mu \mathrm{m}$ (MinerOss ${ }^{\circledR}$; BioHorizons Inc., Birmingham, AL, USA) was used as the sole grafting material. Liquid antibiotic (clindamycin $150 \mathrm{mg}$ / $\mathrm{ml}$ ) was added to the bone graft to minimize the incidence of postoperative infection, using a $1 \mathrm{ml}$ of antibiotic to $2 \mathrm{ml}$ of grafting material ratio. An absorbable collagen material (Collatape ${ }^{\circledR}$; Zimmer Dental, Carlsbad, CA, USA) was placed over the window for hemostatic purposes. Soft tissues were sutured attempting primary closure.

Postoperative instructions were provided after completion of the surgical procedure. Subjects were instructed to take the corticosteroid in decreasing daily doses of 6, 4, and $2 \mathrm{mg}$ starting the day of the surgery. Patients returned for a postoperative follow-up and suture removal at 2 weeks. Thereafter, they were evaluated at 1, 2, 3, 4, and 5 months after maxillary sinus augmentation. Oral hygiene instructions and supragingival deplaquing, if needed, were provided at these visits.

\section{Bone core biopsy harvesting}

At the 5-month follow-up visit, a second CBCT scan utilizing the radiographic guide was obtained. All of the follow-up CBCT scans included the maxilla and maxillary sinuses only. The FOV used was $6 \mathrm{~cm}$ and the machine settings were fixed at $120 \mathrm{kVp}$ and $18.66 \mathrm{mAs}$. An analysis of the grafted area was conducted to assess total bone height achieved and to plan the implant placement surgery, which was performed in all cases between 6 and 7 months after sinus augmentation. Implant placement was performed under local infiltrative anesthesia. The customized radiographic guide was transformed into a surgical guide designed to allow the use of a $3.75 \mathrm{~mm}$ diameter trephine (Salvin Dental Specialties, Inc.), to obtain samples from the exact locations where the radiographic measurements were made. Implant diameter was $\geq 4 \mathrm{~mm}$ in all cases; therefore, in some cases, no drill was utilized after the trephine. Implants were placed in a submerged approach with a minimum insertion torque of $30 \mathrm{~N} / \mathrm{cm}^{2}$ to ensure primary stability, following the manufac- turer's instructions (BioHorizons Internal Implants; BioHorizons Inc., Birmingham, $\mathrm{AL}$, USA).

\section{Histologic preparation}

Immediately after harvesting, biopsies were submerged in a $10 \%$ neutral buffered formalin solution for fixation. Following demineralization in EDTA, cores were dehydrated and embedded in paraffin. Specimens were sectioned following a protocol to accurately obtain circular analyzable samples (Fig. 2). Samples were stained with a conventional hematoxylin-eosin technique and coverslipped for histologic and histomorphometric analysis.

\section{Histomorphometric analysis}

Eight randomized images per sample were captured using a bright field optical microscope with a digital camera (Nikon E800 Light microscope with Diagnostics Spot-RT cooled CCD digital camera, Tokyo, Japan) connected to a computer containing specialized software (Image-Pro Plus 5.0; Media Cybernetics, Bethesda, MD, USA). Vital bone $(\mathrm{VB})$, remaining allograft particle (RA), and non-mineralized tissue (NMT) proportions were measured separately by an experienced examiner (Fig. 3).

\section{Statistical analyses}

All recorded variables $(\mathrm{RBH}, \% \mathrm{VB}, \% \mathrm{RA}$, and $\%$ NMT) were expressed as mean values. The

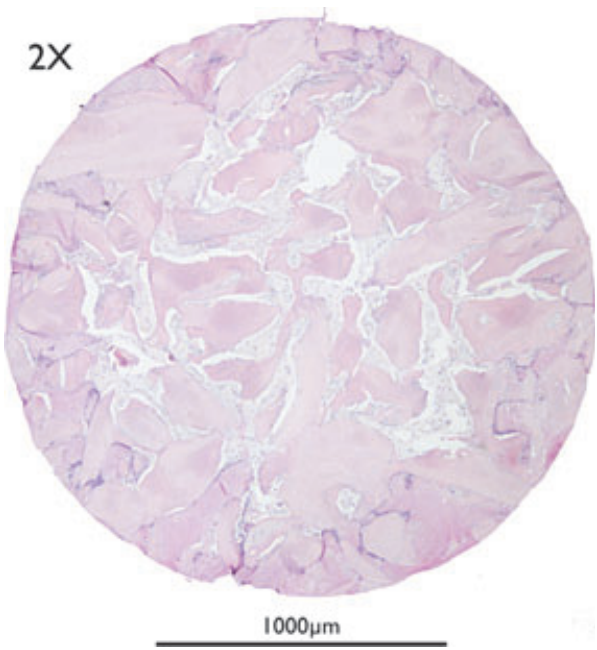

Fig. 2. Histologic section of a representative histologic sample $(H \& E 2 \times)$.

association of $\mathrm{RBH}$ with \% VB and \% RA was quantified as a Pearson's correlation coefficient. RBH was further divided into two categories: $<4$ and $\geq 4 \mathrm{~mm}$. Subsequently, a categorical analysis of correlation was performed for the parameters \% VB and \%RA. The statistical significance of each correlation coefficient estimate was expressed as a $P$-value, where $P<0.05$ was considered statistically significant. The correlation coefficients obtained were adjusted for age and

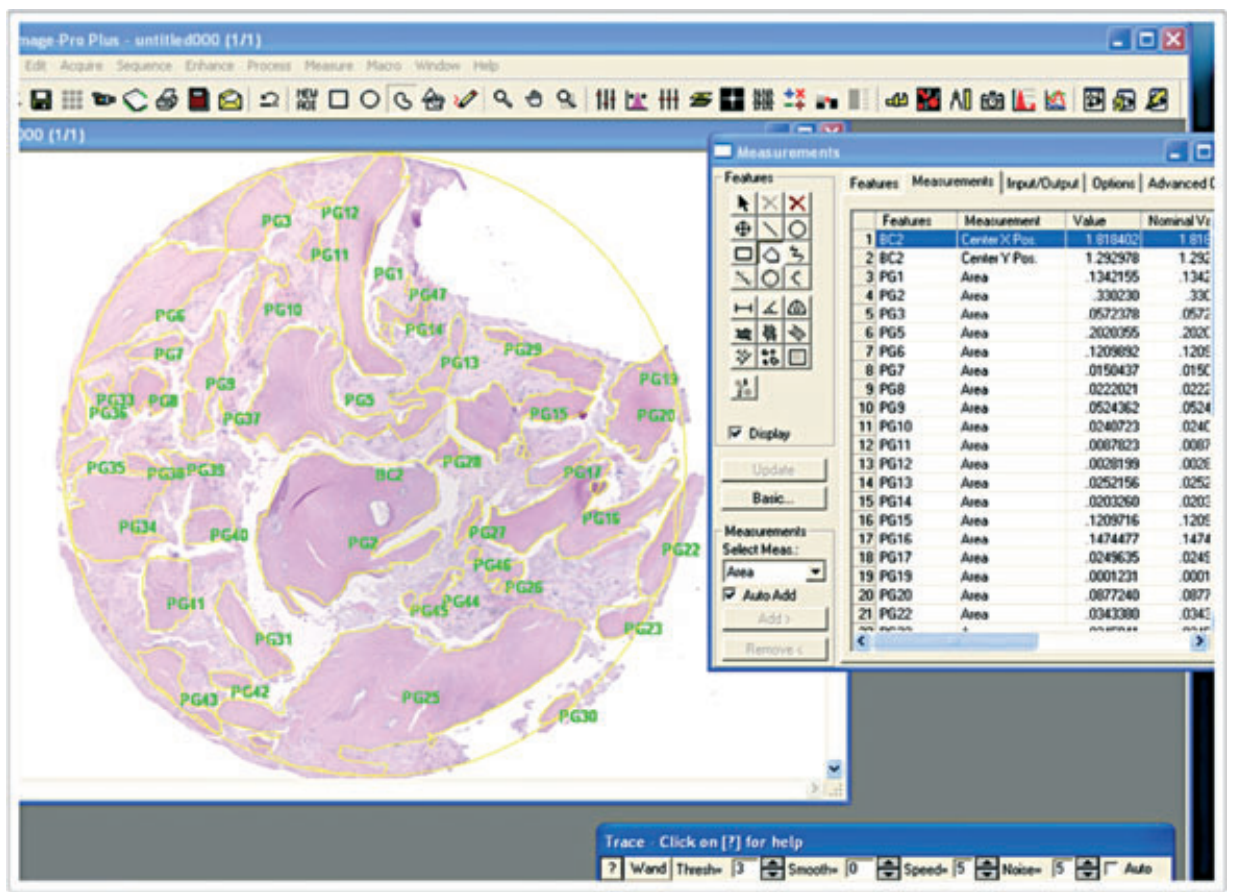

Fig. 3. Computer software was used to perform the histomorphometric analysis. Histologic samples were analyzed in full, comprising the total area. 
gender by calculating partial correlation coefficients.

\section{Results}

\section{Subjects and radiographic findings}

Nine male patients and twelve female patients with a mean age of 57.6 years (ranging from 23 to 69 years) participated in the study. After initial CBCT analysis, four patients were referred for otorhinolaryngological consultation, and all of them were cleared for the procedure. Mean baseline radiographic RBH was $4.25 \pm 1.56 \mathrm{~mm}$, ranging from 1.4 to $6.6 \mathrm{~mm}$. Inter-rater agreement coefficient for RBH was 0.988 , which illustrates a robust positive correlation between measurements made by the two examiners. A total of 21 sinus augmentation procedures were performed. One patient developed a sinusal infection during the initial 2 weeks following bone augmentation. This patient was treated accordingly and the infection was controlled, but was still excluded from the study. All remaining twenty patients were regularly followed-up for the 6-month healing period. In all these cases, augmentation achieved 6 months after sinus augmentation allowed proper implant placement. Mean ridge height achieved after sinus augmentation was $15.9 \pm 2.9 \mathrm{~mm}$, ranging from 12 to $21.6 \mathrm{~mm}$.

\section{Histomorphometric analysis}

Twenty bone biopsies were obtained and sections at a distance of $10 \mathrm{~mm}$ from the alveolar crest were prepared for histomorphometric evaluation. Analysis of hematoxylineosin sections revealed the presence of three tissue components: VB, RA, and NMT. The NMT was compatible with connective tissue presenting two different, coexisting patterns: fibrous and adipose, both in the presence of randomly distributed blood vessels. Newly formed vital bone and remaining allograft particles were basically distinguishable by the presence of osteocytes in the lacunae. Bonelining cells (osteoblasts) were observed over well-organized lamellar bone. Most remaining allograft particles were in intimal contact with newly formed bone, which supports the osteoconductive properties of the material used (Fig. 4). Mean \%VB was $20.47 \pm 18.25$, mean \%RA was $29.04 \pm 24.94$, and a total of $50.47 \pm 12.76$ was found for mean percentage of NMT. Histomorphometric data are expressed in the form of a graphic bar diagram in Fig. 5. In addition, histomorphometric analysis results in relation to $\mathrm{RBH}$ for each sinus are reported in Table 1.

\section{Analysis of correlation}

The correlation between radiographic $\mathrm{RBH}$, $\mathrm{RBH}<4 \mathrm{~mm}$, and $\mathrm{RBH} \geq 4 \mathrm{~mm}$, and both \% $\mathrm{VB}$ and \%RA was analyzed using linear regression analysis (Scatter plot displayed in Fig. 6). No significant correlation between $\mathrm{RBH}$ and \%VB $(r=0.016 ; P=0.951)$, and $\mathrm{RBH}$ and \%RA $(r=0.009 ; \quad P=0.971)$ was found when all data were pooled. Categorical analysis of correlation after stratification of $\mathrm{RBH}$ revealed no statistically significant correlation for any of the associations considered (Table 2). In light of these results, remaining alveolar bone height does not appear to influence the maturation and consolidation of an allograft in the maxillary sinus.

\section{Discussion}

$\mathrm{RBH}$ is a key factor to consider prior to sinus augmentation. For most clinicians,

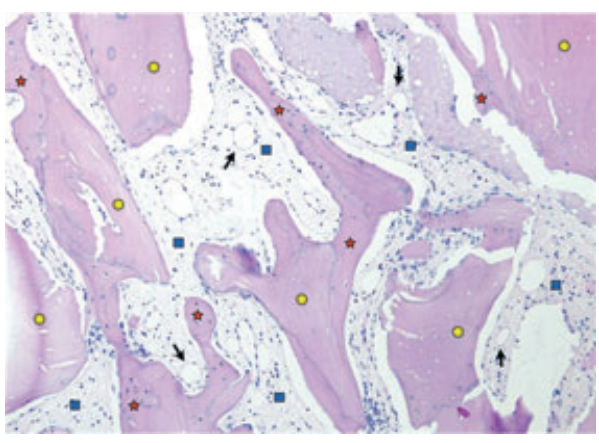

Fig. 4. Detail of a histologic sample showing vital bone (red stars) in intimate contact with remaining allograft particles (yellow circles), embedded in a non-mineralized tissue matrix (blue squares). Black arrows indicate the presence of blood vessels (H\&E 20×).

$\mathrm{RBH}$ determines if implant can be simultaneously placed or not, as RBH may influence the possibility of achieving implant primary stability. The higher the $\mathrm{RBH}$, the better is

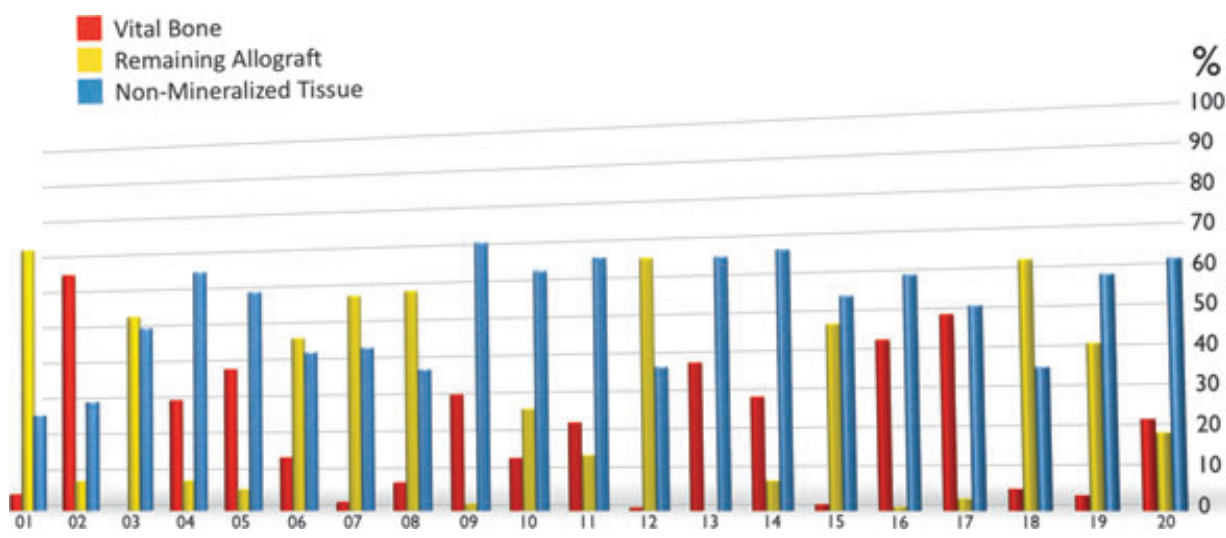

Fig. 5. Diagram showing the total mean values of each element analyzed in the histologic samples, including vital bone (red bars), remaining allograft particle (yellow bars) and non-mineralized connective tissue (blue bars).

Table 1. Residual bone height(RBH) and histomorphometric data values per patient. Corresponding average values of each parameter are shown at the bottom of the table

\begin{tabular}{lllll}
\hline Patient & RBH $(\mathrm{mm})$ & $\% \mathrm{VB}$ & $\% \mathrm{RA}$ & $\% \mathrm{NMT}$ \\
\hline 01 & 3.1 & 4.3 & 70.18 & 25.52 \\
02 & 6.0 & 63.17 & 7.85 & 28.98 \\
03 & 1.4 & 0 & 51.56 & 48.44 \\
04 & 3.8 & 29.21 & 7.83 & 62.96 \\
05 & 1.4 & 37.16 & 5.55 & 57.29 \\
06 & 6.1 & 13.85 & 44.97 & 41.18 \\
07 & 3.7 & 2.21 & 55.67 & 42.12 \\
08 & 4.8 & 7.2 & 56.55 & 36.25 \\
09 & 4.7 & 29.74 & 1.76 & 68.5 \\
10 & 5.7 & 13.33 & 25.82 & 60.85 \\
11 & 3.1 & 22.23 & 13.99 & 63.78 \\
12 & 3.2 & 0.77 & 63.34 & 35.89 \\
13 & 4.9 & 36.89 & 0 & 63.11 \\
14 & 4.9 & 28.16 & 7.36 & 64.48 \\
15 & 3.3 & 1.52 & 45.84 & 52.64 \\
16 & 3.9 & 41.71 & 0.86 & 57.43 \\
17 & 2.4 & 47.58 & 2.86 & 49.56 \\
18 & 6.0 & 5.17 & 60.41 & 34.42 \\
19 & 6.0 & 3.62 & 40 & 56.38 \\
20 & 6.6 & 21.72 & 18.49 & 59.79 \\
Mean \pm SD & $4.25 \pm 1.56$ & $20.47 \pm 18.25$ & $29.04 \pm 24.94$ & $50.47 \pm 12.76$ \\
\hline
\end{tabular}

$\% \mathrm{VB}$, proportion of vital bone; \%RA, remaining allograft particles; $\% \mathrm{NMT}$, non-mineralized tissue. 

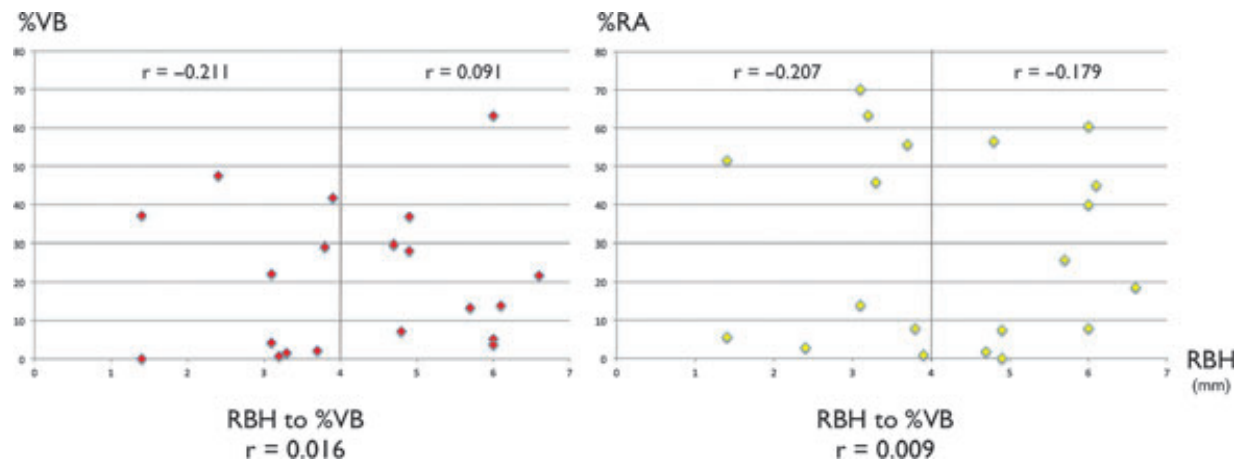

Fig. 6. Scatter plot diagrams illustrating the distribution of $\% \mathrm{VB}$ and $\% \mathrm{RA}$ relative to $\mathrm{RBH}$ with the corresponding correlation coefficient values for pooled $\mathrm{RBH}$ and each $\mathrm{RBH}$ category $(<4$ and $\geq 4 \mathrm{~mm})$, separated by a dotted line.

Table 2. Analysis of correlation values

\begin{tabular}{llrr}
\hline Correlation analysis & & $\%$ VB & $\%$ RA \\
\hline RBH $(n=20)$ & $r$ & 0.016 & 0.009 \\
& $P$-value & 0.951 & 0.971 \\
$\mathrm{RBH}<4 \mathrm{~mm}(n=10)$ & $r$ & -0.211 & 0.207 \\
& $P$-value & 0.617 & 0.622 \\
$\mathrm{RBH} \geq 4 \mathrm{~mm}(n=10)$ & $r$ & 0.091 & -0.179 \\
& $P$-value & 0.830 & 0.672 \\
\hline RBH, residual bone height; \%VB, proportion of vital bone; \%RA, remaining allograft particles. \\
\hline
\end{tabular}

the chance of achieving primary implant stability. Remaining bone heights of 4 and $6 \mathrm{~mm}$ have been proposed as the minimum height that can assure a reliable, simultaneous implant placement. Fenner and coworkers tested the hypothesis that a minimum $\mathrm{RBH}$ is required to obtain better outcomes using an animal model (i.e. minipigs). In their study, the alveolar ridge was surgically remodeled, leaving the crestal aspect intact, to create four different groups (two specimens per group) based on the RBH: 2, 4,6 , and $8 \mathrm{~mm}$. Then, the sinus was grafted and implants were simultaneously placed (six per specimen). Implant stability was assessed using resonance frequency analysis (RFA) at the time of implant placement, 6 months after placement, and 6 months after functional loading. At that time, animals were sacrificed and samples including the implants were retrieved to histologically analyze bone-to-implant contact (BIC) ratio, interthread bone area, peri-implant bone area, and crestal bone resorption. It was concluded that although implant stability and BIC values were worse in the groups with less $\mathrm{RBH}$, the threshold of $5 \mathrm{~mm}$ is not scientifically supported, as osseointegration and implant survival were not significantly affected (Fenner et al. 2009a,b). In a prospective clinical series study using private practice patients, Urban and Lozada compared the success and survival rate of implants placed following a two-stage approach in clinical scenarios with minimal $\mathrm{RBH}$ $(\leq 3.5 \mathrm{~mm})$ and patients with moderate residual crestal bone $(3.5-7 \mathrm{~mm})$. One hundred and fifty-six of 245 implants were placed in the minimal RBH group. Only one implant failed, at the time of abutment placement, and three more failed to meet the success criteria, after 5 years from abutment connection. Overall, implant success and survival rate in this group were $94.1 \%$ and $99.4 \%$, respectively. On the other hand, implant success and survival rate were both $100 \%$ in the moderate RBH group. Interestingly, these differences were not statistically significant (Urban \& Lozada 2010). These results are in agreement with the observations reported in a systematic review aimed at assessing the influence of $\mathrm{RBH}$ on the survival of implants placed simultaneously or delayed after sinus augmentation. Data reviewed from available literature suggest that higher implant survival rate can be expected with higher RBH. Nonetheless, the implant survival rate associated with simultaneous implant placement in the presence of less than $5 \mathrm{~mm}$ of $\mathrm{RBH}$ was found to be very similar to that in cases of $\mathrm{RBH}$ higher than or equal to $5 \mathrm{~mm}$, being $96 \%$ and $99 \%$, respectively (Rios et al. 2009).

Nevertheless, besides implant stability and survival rate, $\mathrm{RBH}$ may also play an important role in the osteogenic potential of the grafted area following maxillary sinus augmentation. New vital bone formation rate, as part of the process of graft consolidation, has been proposed as primarily dependent on the native maxillary bone (Zijderveld et al. 2005). It is important to highlight that bone formation is not exclusively related to the migration and activity of osteogenic cells derived from native bone. The importance of angiogenesis in human bone healing has been greatly emphasized (Carano \& Filvaroff 2003). Microvascular density is essential in graft consolidation, although each biomaterial allows different microvascular colonization (Boeck-Neto et al. 2009; GalindoMoreno et al. 2010). Zerbo et al. (2004) showed, using the sinus augmentation model, that $\beta$-TCP particles lying directly over residual bone of the maxilla were partially or completely replaced by vital bone after 6 months, whereas particles located more apical were still present, suggesting a coronal to apical graft consolidation. Tadjoedin et al. (2003), showed that the front of bone growth primarily originates from the pre-existing native bone surfaces of the maxillary sinus, particularly from the residual alveolar bone. This concept has been recently confirmed by Busenlechner and collaborators, who demonstrated that bone formation is significantly higher in the interface of native bone with different grafting materials, than in deeper areas. However, the gradient of graft consolidation is characteristic of each biomaterial, as reflected by the osteogenic response of the host bone and the degradation profile of the applied bone substitutes (Busenlechner et al. 2009). Our group has previously reported maxillary sinus dimensions influence vital bone formation (Avila et al. 2010a). Similar findings were reported by Artzi et al. (2005), who described that bone area fraction, comparing $\beta$-TCP and anorganic bovine bone, increased from peripheral to deeper zones. In the present study, we aimed at evaluating the influence of $\mathrm{RBH}$ upon the formation of vital bone and the presence of remaining particles using an allograft as a sole grafting material for maxillary sinus augmentation. No significant, either positive or negative, correlation was observed between radiographic $\mathrm{RBH}$ and both $\% \mathrm{VB} \quad\left(\mathrm{R}^{2}=0.248 ; \quad P=0.126\right)$ and \%RA $\left(\mathrm{R}^{2}=-0.065 ; \quad P=0.384\right)$, even when $\mathrm{RBH}$ was stratified into two groups using $4 \mathrm{~mm}$ as the cut-off value. There was a non-linear relationship for both parameters, as it can be observed in the scatter plots included in Fig. 6. Our findings are in agreement with previously discussed available studies indi- 
cating that $\mathrm{RBH}$ may not be such a critical factor to achieve successful outcomes following maxillary sinus augmentation. However, our aim was to evaluate initial maxillary sinus outcomes, instead of long-term implant or implant-supported prostheses success/survival rate. It is possible that implant and implant-supported restorations survival is influenced by the amount of remaining alveolar bone after functional loading. A posthoc power analysis calculation, performed using a specialized software (Faul et al. 2007), revealed that the study has a power of $68 \%$. It is generally acknowledged that $85 \%$ is the standard power required to reject the null hypothesis when it is truly false. This suggests that further studies based on an a priori power analysis with $\mathrm{RBH}$ as the primary outcome, using the information presented in this pilot study, should be conducted to shed more light on this interesting topic. Furthermore, another limitation of this study stems from the interindividual variability in histomorphometric outcomes, particularly in terms of vital bone and remaining allograft particle, as reflected in Table 1 and Fig. 5. Despite our efforts to enroll a homogeneous patient population, based on the inclusion and exclusion criteria, this marked variability could be explained by individual healing potential, and other confounders such as the occurrence of concomitant respiratory tract pathology (e.g. common viral cold) during the healing period.

The use of prophylactic antibiotics prior to sinus augmentation procedures is a controversial topic. In general, the use of antibiotic prophylaxis is generally limited to those cases in which there is a concomitant local infection and when patients with comorbid conditions or immunodepression are treated. The rationale is to avoid pharmacologic aller-

\section{References}

Artzi, Z., Kozlovsky, A., Nemcovsky, C.E. \& Weinreb, M. (2005) The amount of newly formed bone in sinus grafting procedures depends on tissue depth as well as the type and residual amount of the grafted material. Journal of Clinical Periodontology 32: 193-199.

Avila, G., Neiva, R., Misch, C.E., Galindo-Moreno, P., Benavides, E., Rudek, I. \& Wang, H.L. (2010a) Clinical and histologic outcomes after the use of a novel allograft for maxillary sinus augmentation: a case series. Implant Dentistry 19: 330 341.

Avila, G., Wang, H.L., Galindo-Moreno, P., Misch, C.E., Bagramian, R.A., Rudek, I., Benavides, E., Moreno-Riestra, I., Braun, T. \& Neiva, R. (2010b) The influence of the bucco-palatal distance on sinus augmentation outcomes. Journal of Periodontology 81: 1041-1050. gic reactions and antibiotic resistances. Interestingly, Powell et al. (2005), showed that patients who received antibiotics exhibited a higher rate of infection than patients who had no antibiotic coverage, although the difference was not statistically significant. In the population they studied, no patient who underwent maxillary sinus augmentation $(\mathrm{n}=15)$ developed an infection. However, 'the prophylactic use of systemic and local antibiotics and glucocorticosteroids can reduce the risk of infection' (Misch 1992). This recommendation finds a clear indication in the event of Schneiderian membrane perforation, since a retrograde bacterial colonization from the respiratory tract may lead to an infection of the grafted area. Given the impossibility of predicting in which cases that complication is going to occur, to provide a standardized treatment to all patients participating in this controlled study, we followed the pharmacologic protocol proposed by Resnik \& Misch (2008). Finally, to our knowledge, there is limited information available with regard to impact of local antibiotic application in the healing following oral bone grafting and conflicting results have been reported (Kim et al. 2004; Oghli \& Steveling 2010). We consider that, although possible, it is unlikely that the use of liquid clindamycin in conjunction with the bone grafting material has a determining influence in bone healing and histomorphometric values of samples harvested after a 6-month healing period.

\section{Conclusions}

Our findings suggest that remaining alveolar bone height does not influence the matura- tion and consolidation of an allograft 6 months after maxillary sinus augmentation.

Acknowledgements: This study was partially supported by BioHorizons Inc., ICOI Implant Dentistry Research and Education Foundation Research Grant, and the University of Michigan Periodontal Graduate Student Research Fund. The authors thank Hector F. Rios (Associate Professor, Department of Periodontics and Oral Medicine, University of Michigan School of Dentistry, Ann Arbor, MI, USA), Mary Layher /Graduate Periodontics clinical research coordinator, Department of Periodontics and Oral Medicine, University of Michigan School of Dentistry, Ann Arbor, MI, USA), Cynthia Tsoukalas, Erika Mischung, and Amy Lawson/Clinical assistants, Department of Periodontics and Oral Medicine, University of Michigan School of Dentistry, Ann Arbor, MI, USA), and Lori Jackson (Administrative staff, Department of Periodontics and Oral Medicine, University of Michigan School of Dentistry, Ann Arbor, MI, USA) for their help in the development of the study. Conflict of interest: Drs. Neiva and Wang declare having received financial support for research and lecture fees from organizations that may either gain or lose from the publication of this paper. The other authors declare having no conflict of interest with any of the companies or products listed in this study.
Boeck-Neto, R.J., Artese, L., Piattelli, A., Shibli, J. A., Perrotti, V., Piccirilli, M. \& Marcantonio, E., Jr (2009) VEGF and MVD expression in sinus augmentation with autologous bone and several graft materials. Oral Diseases 15: 148-154.

Busenlechner, D., Huber, C.D., Vasak, C., Dobsak, A., Gruber, R. \& Watzek, G. (2009) Sinus augmentation analysis revised: the gradient of graft consolidation. Clinical Oral Implants Research 20: $1078-1083$.

Carano, R.A. \& Filvaroff, E.H. (2003) Angiogenesis and bone repair. Drug Discovery Today 8: 980989

Faul, F., Erdfelder, E., Lang, A.G. \& Buchner, A. (2007) G*Power 3: a flexible statistical power analysis program for the social, behavioral, and biomedical sciences. Behavior Research Methods 39: 175-191.
Fenner, M., Vairaktaris, E., Fischer, K., Schlegel, K. A., Neukam, F.W. \& Nkenke, E. (2009a) Influence of residual alveolar bone height on osseointegration of implants in the maxilla: a pilot study. Clinical Oral Implants Research 20: 555559.

Fenner, M., Vairaktaris, E., Stockmann, P., Schlegel, K.A., Neukam, F.W. \& Nkenke, E. (2009b) Influence of residual alveolar bone height on implant stability in the maxilla: an experimental animal study. Clinical Oral Implants Research 20: 751755.

Galindo-Moreno, P., Padial-Molina, M., FernandezBarbero, J.E., Mesa, F., Rodriguez-Martinez, D. \& O’Valle, F. (2010) Optimal microvessel density from composite graft of autogenous maxillary cortical bone and anorganic bovine bone in sinus augmentation: influence of clinical vari- 
ables. Clinical Oral Implants Research 21: 221227.

Kim, S.G., Chung, T.Y., Kim, M.S. \& Lim, S.C. (2004) The effect of high local concentrations of antibiotics on demineralized bone induction in rats. Journal of Oral and Maxillofacial Surgery 62: 708-713.

Levin, L., Herzberg, R., Dolev, E. \& Schwartz-Arad, D. (2004) Smoking and complications of onlay bone grafts and sinus lift operations. International Journal of Oral and Maxillofacial Implants 19: 369-373.

Mardinger, O., Nissan, J. \& Chaushu, G. (2007) Sinus floor augmentation with simultaneous implant placement in the severely atrophic maxilla: technical problems and complications. Journal of Periodontology 78: 18721877.

Misch, C.M. (1992) The pharmacologic management of maxillary sinus elevation surgery. Journal of Oral Implantology 18: 15-23.

Misch, C., Resnik, R.R. \& Misch-Dietsh, F. (2009) Maxillary sinus anatomy, pathology, and graft surgery. In: Misch, C., ed. Contemporary Implant Dentistry, 3rd edition, 903-974. St. Louis: Mosby Elsevier.

Nkenke, E. \& Stelzle, F. (2009) Clinical outcomes of sinus floor augmentation for implant placement using autogenous bone or bone substitutes: a systematic review. Clinical Oral Implants Research 20(Suppl. 4): 124-133.

Oghli, A.A. \& Steveling, H. (2010) Ridge preservation following tooth extraction: a comparison between atraumatic extraction and socket seal surgery. Quintessence International Dental Journal 41: 605-609.

O'Leary, T.J., Drake, R.B. \& Naylor, J.E. (1972) The plaque control record. Journal of Periodontology 43: 38 .

Peleg, M., Garg, A.K. \& Mazor, Z. (2006) Predictability of simultaneous implant placement in the severely atrophic posterior maxilla: a 9-year longitudinal experience study of 2132 implants placed into 731 human sinus grafts. International Journal of Oral and Maxillofacial Implants 21: 94 102.

Peleg, M., Mazor, Z., Chaushu, G. \& Garg, A.K. (1998) Sinus floor augmentation with simultaneous implant placement in the severely atrophic maxilla. Journal of Periodontology 69: 1397-1403.

Peleg, M., Mazor, Z. \& Garg, A.K. (1999) Augmentation grafting of the maxillary sinus and simultaneous implant placement in patients with 3 to $5 \mathrm{~mm}$ of residual alveolar bone height. International Journal of Oral and Maxillofacial Implants 14: 549-556.

Powell, C.A., Mealey, B.L., Deas, D.E., McDonnell, H.T. \& Moritz, A.J. (2005) Post-surgical infections: prevalence associated with various periodontal surgical procedures. Journal of Periodontology $\mathbf{7 6}$ : 329-333.

Resnik, R.R. \& Misch, C. (2008) Prophylactic antibiotic regimens in oral implantology: rationale and protocol. Implant Dentistry 17: 142-150.

Rios, H.F., Avila, G., Galindo-Moreno, P. \& Wang, H.L. (2009) The Influence of Remaining Alveolar Bone Upon Lateral Window Sinus Augmentation Implant Survival. Implant Dentistry. 18: 402-412.
Rodriguez, A., Anastassov, G.E., Lee, H., Buchbinder, D. \& Wettan, H. (2003) Maxillary sinus augmentation with deproteinated bovine bone and platelet rich plasma with simultaneous insertion of endosseous implants. Journal of Oral and Maxillofacial Surgery 61: 157-163.

Tadjoedin, E.S., de Lange, G.L., Bronckers, A.L., Lyaruu, D.M. \& Burger, E.H. (2003) Deproteinized cancellous bovine bone (Bio-Oss) as bone substitute for sinus floor elevation. A retrospective, histomorphometrical study of five cases. Journal of Clinical Periodontology 30: 261-270.

Urban, I.A. \& Lozada, J.L. (2010) Implants placed in augmented sinuses with minimal and moderate residual crestal bone: results after 1 to 5 years. International Journal of Oral and Maxillofacial Implants 25: 1203-1212.

Wang, H.L. \& Katranji, A. (2008) ABC sinus augmentation classification. International Journal of Periodontics and Restorative Dentistry 28: 383-389.

Zerbo, I.R., Zijderveld, S.A., de Boer, A., Bronckers, A.L., de Lange, G., ten Bruggenkate, C.M. \& Burger, E.H. (2004) Histomorphometry of human sinus floor augmentation using a porous beta-tricalcium phosphate: a prospective study. Clinical Oral Implants Research 15: 724-732.

Zijderveld, S.A., Zerbo, I.R., van den Bergh, J.P., Schulten, E.A. \& ten Bruggenkate, C.M. (2005) Maxillary sinus floor augmentation using a betatricalcium phosphate (Cerasorb) alone compared to autogenous bone grafts. International Journal of Oral and Maxillofacial Implants 20: 432-440. 